\title{
Large Deformation Diffeomorphic Registration of Diffusion-Weighted Images
}

\author{
Pei Zhang ${ }^{1}$, Marc Niethammer ${ }^{2}$, Dinggang Shen ${ }^{1}$, and Pew-Thian Yap ${ }^{1, *}$ \\ 1 Department of Radiology, \\ 2 Department of Computer Science, \\ Biomedical Research Imaging Center (BRIC), \\ The University of North Carolina at Chapel Hill, USA \\ peizhang@email.unc.edu, mn@cs.unc.edu, \{ptyap, dgshen\}@med.unc.edu
}

\begin{abstract}
Registration of Diffusion-weighted imaging (DWI) data emerges as an important topic in magnetic resonance (MR) image analysis. As existing methods are often designed for specific diffusion models, it is difficult to fit to the registered data different models other than the one used for registration. In this paper we describe a diffeomorphic registration algorithm for DWI data in a large deformation setting. Our method generates spatially normalized DWI data and it is thus possible to fit various diffusion models after registration for comparison purposes. Our algorithm includes (1) a reorientation component, where each diffusion profile (DWI signal as a function on a unit sphere) is decomposed, reoriented and recomposed to form the orientation-corrected DWI profile, and (2) a large deformation diffeomorphic registration component to ensure one-to-one mapping in a large-structural-variation scenario. In addition our algorithm uses a geodesic shooting mechanism to avoid the huge computational resources that are needed to register high-dimensional vector-valued data. We also incorporate into our algorithm a multi-kernel strategy where anatomical structures at different scales are considered simultaneously during registration. We demonstrate the efficacy of our method using in vivo data.
\end{abstract}

\section{Introduction}

DWI registration presents a direct way of establishing correspondences for white matter micro-structures, which are often elusive in anatomical scans, such as $\mathrm{T}_{1}$ - and $\mathrm{T}_{2}$ weighted images. As it is required to deal with both spatial alignment of macro-structures and reorientation of local angular structures, DWI registration is more challenging to develop than traditional scalar-based image registration.

DWI data are often acquired in up to hundreds of diffusion-sensitizing gradient directions so as to precisely delineate local angular structures. Various diffusion models are often fitted to the acquired data for analytical purposes. However, analysis can not yet be performed without aligning similar structures across different subjects. To this end, a number of registration algorithms have thus been developed. Geng et al. [1] used

\footnotetext{
* Corresponding author. This work was supported in part by a UNC start-up fund, NSF grants (EECS-1148870 and EECS-0925875) and NIH grants (EB006733, EB008374, EB009634, MH088520, NIHM 5R01MH091645-02, and AG041721).
} 
a spherical harmonic $(\mathrm{SH})$ representation of orientation distribution functions (ODFs) to guide registration. Yap et al. [2] developed a hierarchical registration scheme where the alignment is refined using features extracted from a SH-based representation with gradually increasing order. Raffelt et al. [3] utilized a subject-template-symmetric diffeomorphic framework to align fiber orientation distribution (FOD) fields. Hong et al. [4] performed registration with the help of $\mathrm{T}_{2}$-weighted images and applied the resulting deformation fields to the diffusion-weighted images with re-transformation-taking into account rotation, scaling, and shearing effects of the spatial transformation of the FOD. Du et al. [5] designed a large deformation framework to register the ODF data.

However, all of the above methods are designed for specific diffusion models, which makes it difficult to fit other models to the registered data. In this paper we describe a method that is able to generate spatially normalized DWI data so that one can fit any diffusion model after registration. Key highlights of our method include:

1. DWI Reorientation: Our method can directly reorient DWI diffusion signal profiles.

2. Large Deformation: We use a large deformation diffeomorphic metric mapping (LDDMM) framework [6] to tackle large structural variations. Spatial image alignment is achieved by optimizing over a spatio-temporally varying velocity field.

3. Geodesic Shooting: A major problem with the LDDMM algorithm [6] is the large memory consumption. This is often aggravated for vector-valued and high dimensional DWI data. We thus use a geodesic shooting algorithm [7] to avoid the storage of the entire series of velocity fields, so that only an initial image and an initial momentum are needed to parameterize the full deformation path.

4. Multi-Kernel: We use multiple Gaussian kernels [8] to simultaneously register anatomical structures at different scales.

Works on registering the raw DWI data are few. To the best of our knowledge, the only closest work is that of Dhollander et al. [9], where they achieved the goal by using SHs [3] as well as a diffeomorphic demons algorithm [10]. Our method differs fundamentally from theirs in three aspects: (1) We achieve reorientation by using Watson distributions instead of SHs. This avoids the computational complexity of SHs as well as the loss of sharp directional information when SH basis functions of insufficient order are used; (2) Our method can work with single-shell DWI data, whereas their method requires multi-shell data acquisition, which might be clinically infeasible; (3) Our method explicitly considers large deformation.

\section{Methodology}

Below we will first describe the approach to reorienting DWI data in $Q$-space. We will then focus on the simplified shooting algorithm used for registration. Finally, a summary of the proposed method will be given.

\subsection{Reorientation of DWI Data}

To reorient the DWI data in $Q$-space we first decompose the diffusion signal profile into a series of fiber basis functions (FBFs), which are based on the Watson distribution function [11]. We then apply a local transformation, computed from the estimated 


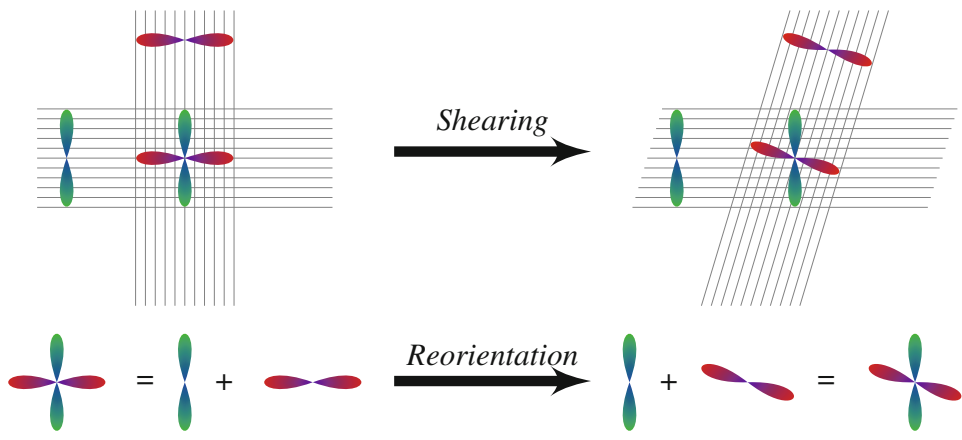

Fig. 1. Two fiber populations (gray lines) are shown together with their individual diffusion signal profiles. When the two fiber populations cross each other, the acquired diffusion signal profile is a combination of the responses from both fiber populations. As each fiber population transforms differently with respect to a local transformation (horizontal shearing in this example), the profile at the crossing should be decoupled, reoriented individually, and then recombined to form a reoriented diffusion signal profile.

map, to reorient each FBF independently. Finally, we recompose the reoriented FBFs to obtain the orientation-rectified DWI profile. See Fig. 1 for an illustration.

Diffusion Profile Modeling. Let $S\left(\mathbf{q}_{i}\right)$ be the diffusion signal measured in direction $\mathbf{q}_{i}(i=1, \ldots, M)$. Our goal is to represent $S\left(\mathbf{q}_{i}\right)$ in terms of a series of FBFs. As we use a set of Watson distributions [11] to realize the FBFs, we can write

$$
S\left(\mathbf{q}_{i}\right)=w_{0} f_{0}+\sum_{j=1}^{N} w_{j} f\left(\mathbf{q}_{i} \mid \boldsymbol{\mu}_{j}, \kappa\right), \quad \kappa<0,
$$

where $f(\mathbf{q} \mid \boldsymbol{\mu}, \kappa)=C(\kappa) \exp \left(\kappa\left(\boldsymbol{\mu}^{\mathrm{T}} \mathbf{q}\right)^{2}\right)$ is the probability density function of the Watson distribution [11], $\mathbf{q}$ and $\boldsymbol{\mu}$ are unit vectors indicating the diffusion gradient direction and the mean orientation respectively, $\kappa$ is a constant, and $C(\kappa)$ is the normalization factor. $w_{j}$ is the weight associated with each FBF $f(\cdot) . f_{0} \equiv C(0)$ is a constant representing the isotropic diffusion component. Given the diffusion signal profile $\mathbf{S}=\left[S\left(\mathbf{q}_{1}\right), \ldots, S\left(\mathbf{q}_{M}\right)\right]^{\mathrm{T}}$, we have $\mathbf{S}=\mathbf{F} \mathbf{w}$, where $\mathbf{w}=\left[w_{0}, w_{1}, \ldots, w_{N}\right]^{\mathrm{T}}$ and

$$
\mathbf{F}=\left[\begin{array}{cccc}
f_{0} & f\left(\mathbf{q}_{1} \mid \boldsymbol{\mu}_{1}, \kappa\right) & \cdots & f\left(\mathbf{q}_{1} \mid \boldsymbol{\mu}_{N}, \kappa\right) \\
\vdots & \vdots & \ddots & \vdots \\
f_{0} & f\left(\mathbf{q}_{M} \mid \boldsymbol{\mu}_{1}, \kappa\right) & \cdots & f\left(\mathbf{q}_{M} \mid \boldsymbol{\mu}_{N}, \kappa\right)
\end{array}\right] .
$$

Since typically, $M<N+1$, we have a set of underdetermined linear equations. We solve this using a $L_{1}$ regularized least-squares solver with a non-negative constraint. The reader is referred to [12] for details of the algorithm and evaluation.

Transformation and Recomposition. To reorient the direction of each FBF, $\boldsymbol{\mu}_{j}$, we apply a local affine transformation $\mathbf{A}$ estimated from the map resulting from registration, i.e. $\boldsymbol{\mu}_{j}^{\prime}=\mathbf{A} \boldsymbol{\mu}_{j} /\left\|\mathbf{A} \boldsymbol{\mu}_{j}\right\|$. A matrix of rotated FBFs, $\mathbf{F}^{\prime}$, can be then computed 
based on $\boldsymbol{\mu}_{j}^{\prime}$. The transformed DWI signal $\mathbf{S}^{\prime}$ is finally computed as $\mathbf{S}^{\prime}=\mathbf{F}^{\prime} \mathbf{w}$. Note that the isotropic component is not rotated.

\subsection{A Simplified Geodesic Shooting Algorithm}

We now describe the registration method for a pair of DWI data. To avoid the computational complexity of a full adjoint shooting method [13], we follow the simplified shooting approach [7]. However, we modify it to allow for a gradient descent directly on the initial Hamiltonian momentum (the co-adjoint variable to the transported image, instead of the vector-valued momentum).

Below we use $I$ to represent a vector-valued image of a diffusion profile $\mathbf{S}$ at each voxel location, and $I^{i}$ to denote the $i$-th channel of $I$. Let $I_{0}$ be the source image and $I_{1}$ be the target image. Our goal is to minimize

$E(v, I)=\frac{1}{2} \int_{0}^{1}\|v\|_{V}^{2} \mathrm{~d} t+\frac{1}{\sigma^{2}} \sum_{i=1}^{M}\left\|I^{i}(1)-I_{1}^{i}\right\|_{L^{2}}^{2}$, s.t. $I_{t}^{i}+\nabla\left(I^{i}\right)^{\mathrm{T}} v=0, I^{i}(0)=I_{0}^{i}$,

where $v$ is the sought-for time-dependent velocity field, $\sigma>0$ is a constant and $\|v\|_{V}^{2}=$ $\left\langle L^{\dagger} L v, v\right\rangle_{L^{2}}$, where $L$ is a proper differential operator. Instead of defining $L$ we define a desired smoothing kernel $K=\left(L^{\dagger} L\right)^{-1}$. We use a multi-Gaussian kernel [8] to introduce a natural multi-resolution property to the solution and to provide an intuitive way of parameter tuning based on the desired scales that should be captured by the registration. Note that we run our algorithm with multiple iterations to minimize (2). In each iteration, $I_{0}$ is spatially transformed and reoriented using the map estimated in the previous iteration (see Sect.2.3 for details).

The minimization of (2) leads to the following optimality and boundary conditions:

$$
\begin{cases}I_{t}^{i}+\nabla\left(I^{i}\right)^{\mathrm{T}} v & =0, I^{i}(0)=I_{0}^{i} \\ -p_{t}^{i}-\operatorname{div}\left(p^{i} v\right) & =0, p^{i}(1)=-\frac{2}{\sigma^{2}}\left(I^{i}(1)-I_{1}^{i}\right) \\ L^{\dagger} L v+\sum_{i=1}^{M} p^{i} \nabla I^{i} & =0\end{cases}
$$

Note that $\nabla_{v} E=L^{\dagger} L v+\sum_{i=1}^{M} p^{i} \nabla I^{i}$. Hence, instead of solving (3) as a boundary value problem [6] we follow a simplified shooting approach [7], performing the gradient descent only for $t=0$. In contrast to [7], here we perform the gradient descent directly on the $\left\{p^{i}(0)\right\}$ by pulling the final conditions $\left\{p^{i}(1)\right\}$ back to $t=0$. This can be accomplished by computing a backward map (from $t=1$ to $t=0$ ) on the fly during a forward integration (from $t=0$ to $t=1$ ). To obtain the gradient with respect to these momentum variables note that at convergence (or on a geodesic in general) for all times $L^{\dagger} L v+\sum_{i=1}^{M} p^{i} \nabla I^{i}=0$. Therefore at $t=0$

$$
L^{\dagger} L \delta v(0)+\sum_{i=1}^{M} \delta p^{i}(0) \nabla I^{i}(0)=0
$$

because $I(0)=I_{0}$ is known. 
In the vector-valued version of the standard LDDMM scheme [6] (which, given a velocity field $v$ uses a forward sweep for $I^{i}$ and a backward sweep for $p^{i}$ ) the Hilbert gradient at $t=0$ is computed as

$$
\nabla_{v(0)} E=v(0)+\left(L^{\dagger} L\right)^{-1}\left(\sum_{i=1}^{M} \hat{p}^{i}(0) \nabla I^{i}(0)\right),
$$

where $\hat{p}^{i}(0)$ is the adjoint at $t=0$ obtained after the forward sweep for $I^{i}$, which allows the computation of $\hat{p}^{i}(1)$ followed by a backward sweep for $p^{i}$. Since $v(0)=$ $-\left(L^{\dagger} L\right)^{-1}\left(\sum_{i=1}^{M} p^{i}(0) \nabla I^{i}(0)\right)$ is the initial velocity given the current initial momentum $p^{i}(0)$ the gradient can be rewritten as

$$
\nabla_{v(0)} E=\left(L^{\dagger} L\right)^{-1}\left[\sum_{i=1}^{M}\left(\hat{p}^{i}(0)-p^{i}(0)\right) \nabla I^{i}(0)\right] .
$$

Substituting into (4) we obtain

$$
\sum_{i=1}^{M} \delta p^{i}(0) \nabla I^{i}(0)=\sum_{i=1}^{M}\left(p^{i}(0)-\hat{p}^{i}(0)\right) \nabla I^{i}(0) .
$$

Since this needs to hold for any initial image $I(0)$ it follows that $\nabla_{p^{i}(0)} E=p^{i}(0)-$ $\hat{p}^{i}(0)$. Given the (on-the-fly) computed map $\Phi$ which maps $t=1$ to $t=0$ the gradient is then $\nabla_{p^{i}(0)} E=p^{i}(0)-|D \Phi| \hat{p}^{i}(1) \circ \Phi$.

\subsection{Summary of the Approach}

We first use the method described in Sect.2.1 to decompose both $I_{0}$ and $I_{1}$, and then run the above geodesic-shooting LDDMM to iteratively transform and reorient $I_{0}$. Specifically, we first estimate a global affine transformation $\mathbf{A}_{g}$ between the anisotropy images of $I_{0}$ and $I_{1}$. And then at each iteration, we (1) reconstruct both $I_{0}$ and $I_{1}$ with a decreasing $\kappa$ and an increasing number of diffusion directions. $I_{0}$ is reconstructed using the FBFs reoriented with the map estimated in the previous iteration together with $\mathbf{A}_{g}$, while $I_{1}$ is reconstructed using the original FBFs with an identity map; (2) weight each reconstructed image using the associated anisotropy image; (3) estimate the map between the weighted images; and (4) compose the resulting map with the one estimated in the previous iteration. At the end of the registration we will obtain the final map between $I_{0}$ and $I_{1}$ as well as a transformed and reoriented source image $I_{0}^{\prime}$.

\section{Experiments}

The DWI data were acquired from 11 adults using a Siemens 3T TIM Trio MR Scanner with an EPI sequence. Diffusion gradients were applied in 120 non-collinear directions with diffusion weighting $b=2000 \mathrm{~s} / \mathrm{mm}^{2}$. The imaging matrix is $128 \times 128$ with rectangular FOV of $256 \times 256 \mathrm{~mm}^{2} .80$ contiguous slices with a slice thickness of $2 \mathrm{~mm}$ cover the whole brain. 
We randomly chose a subject and used the associated data as the target image, and used the rest of the data as the source images. We computed the anisotropy images of all data and used them to estimate a set of affine transformations $\left\{\mathbf{A}_{g}\right\}$. We then warped each source image to the target image using the associated $\mathbf{A}_{g}$ and computed the mean image of the anisotropy images of the warped source (Fig.2 $\mathrm{b}$ ). This blurred mean image implies that the DWI data cannot be well aligned by affine transformation.

We then used our method (3 iterations) to register each source image to the target image. After registration, we reconstructed each source in the original 120 directions using the associated $\mathbf{A}_{g}$ together with the resulting map. Averaging the anisotropy images across the subjects leads to the mean image shown in Fig.2 d. Repeating the above process with the map generated in the first iteration gives the mean in Fig. 2k. We found that our method significantly outperforms affine registration by producing a much crisper mean. Further improvement can be achieved by running the registration multiple times.

To quantify the comparison, we computed the RMS error between the vector-valued voxels at corresponding positions. This was done between the target and each source image, warped either using $\left\{\mathbf{A}_{g}\right\}$ or the map estimated by our method. Averaging the resulting RMS error images across the subjects leads to the mean images shown in Fig. $2 \mathrm{e}-\mathrm{g}$. The mean and standard deviation (s.d.) of these mean images are $13.5 \pm 6.2$ for affine registration, $11.0 \pm 4.8$ for the first iteration, and $10.3 \pm 4.6$ for the final iteration.

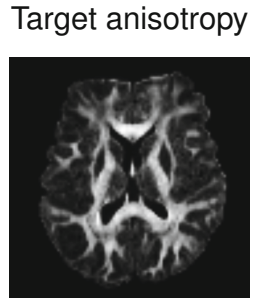

(a)

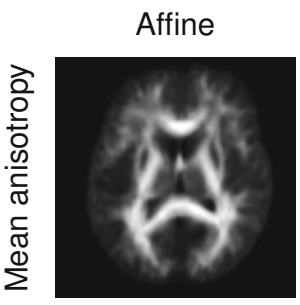

(b)
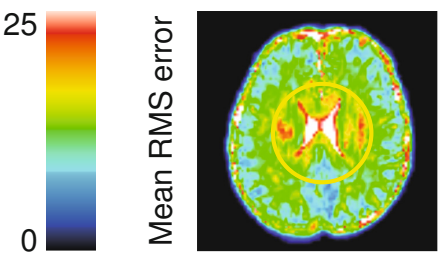

(e)

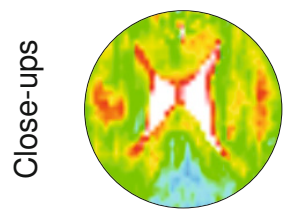

(h)
Initial iteration

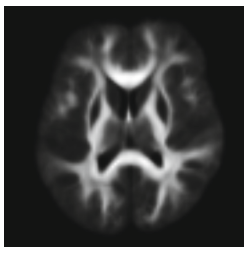

(c)

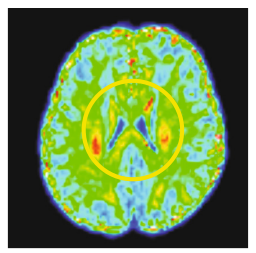

(f)

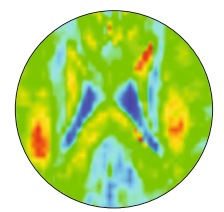

(i)
Final iteration

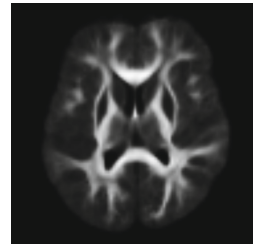

(d)

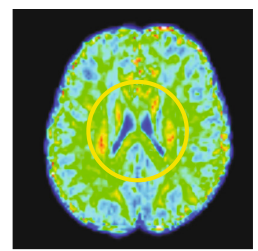

(g)

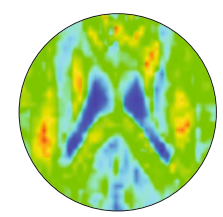

(j)

Fig. 2. Comparison of registration accuracy between affine registration and our method 

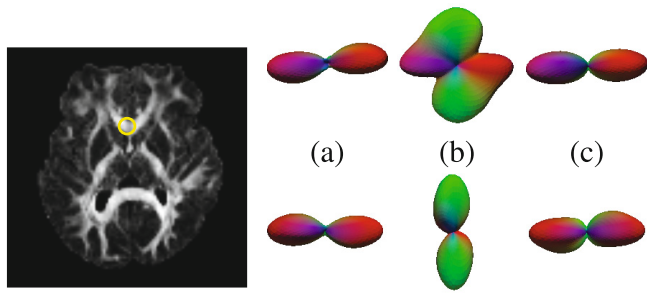

(d)

(e)

(f)

Fig. 3. A region of interest is indicated by the yellow circle in the left figure and some typical ODFs associated with this region are given on the right. (a) and (d): the ODFs of the target image; (b) and (e): the ODFs of the mean image generated using affine registration; (c) and (f): the ODFs of the mean image generated using our method.
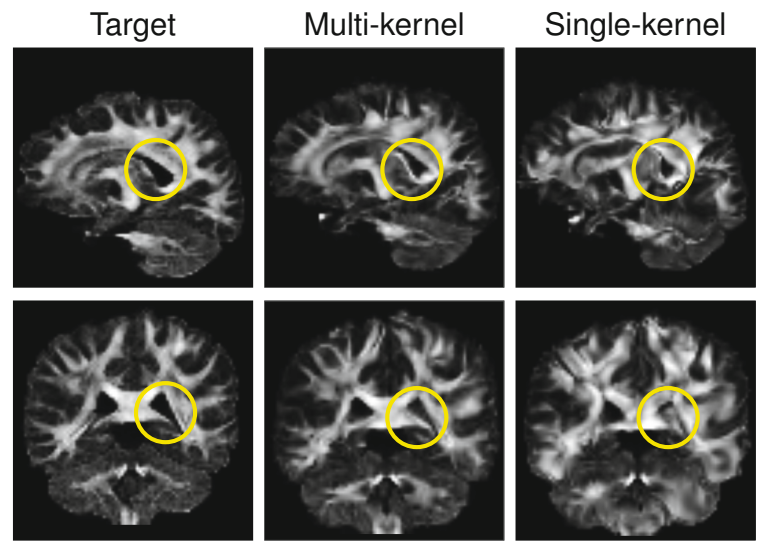

Fig. 4. Comparison of registration results given by multiple Gaussian kernels and a single kernel

We also labeled a set of salient landmark points (around the lateral ventricles) on the anisotropy images. For affine registration we used $\left\{\mathbf{A}_{g}\right\}$ to warp the landmarks of each source to the target space, and computed the mean and s.d. of the Euclidean distances (in $\mathrm{mm}$ ) between the warped landmarks and the corresponding landmarks on the target. For our method this was done by using $\left\{\mathbf{A}_{g}\right\}$ as well as the resulting maps. The results for affine registration, the first iteration and the final iteration are 7.0 $\pm 3.0,4.4 \pm 2.4$ and $3.6 \pm 2.2$.

The results from these two quantitative comparisons are in agreement with our observations based on the mean anisotropy images.

Figure 3 shows that at voxel level the alignment of the DWI profiles across subjects can greatly benefit from registration using the proposed method. The ODFs of the average DWI data can deviate significantly when registration is inaccurate.

To demonstrate the advantage of using multiple Gaussian kernels, we repeated the above experiment by using only one Gaussian kernel. Figure 4 clearly shows that a single kernel is unlikely to capture all shape variations that are present in the data. 


\section{Concluding Remarks}

We have described a method for direct registration of DWI data. Our method is capable of producing spatially normalized DWI data, based on which any diffusion model can be fitted for comparison purposes. Future work includes unifying the registration and reorientation steps for further improvement on alignment accuracy as well as comparing the performance of different diffusion models within the proposed framework.

\section{References}

1. Geng, X., Ross, T.J., Zhan, W., Gu, H., Chao, Y.-P., Lin, C.-P., Christensen, G.E., Schuff, N., Yang, Y.: Diffusion MRI Registration Using Orientation Distribution Functions. In: Prince, J.L., Pham, D.L., Myers, K.J. (eds.) IPMI 2009. LNCS, vol. 5636, pp. 626-637. Springer, Heidelberg (2009)

2. Yap, P.T., Chen, Y., An, H., Yang, Y., Gilmore, J.H., Lin, W., Shen, D.: SPHERE: Spherical harmonic elastic registration of HARDI data. NeuroImage 55(2), 545-556 (2011)

3. Raffelt, D., Tournier, J.D., Fripp, J., Crozier, S., Connelly, A., Salvado, O.: Symmetric diffeomorphic registration of fibre orientation distributions. NeuroImage 56(3), 1171-1180 (2011)

4. Hong, X., Arlinghaus, L., Anderson, A.: Spatial normalization of the fiber orientation distribution based on high angular resolution diffusion imaging data. Magnetic Resonance in Medicine 61(6), 1520-1527 (2009)

5. Du, J., Goh, A., Qiu, A.: Diffeomorphic metric mapping of high angular resolution diffusion imaging based on riemannian structure of orientation distribution functions. IEEE Transactions on Medical Imaging 31(5), 1021-1033 (2012)

6. Beg, M.F., Miller, M.I., Trouvé, A., Younes, L.: Computing large deformation metric mappings via geodesic flows of diffeomorphisms. International Journal of Computer Vision 61(2), 139-157 (2005)

7. Ashburner, J., Friston, K.J.: Diffeomorphic registration using geodesic shooting and Gauss Newton optimisation. NeuroImage 55(3), 954-967 (2011)

8. Risser, L., Vialard, F.X., Wolz, R., Murgasova, M., Holm, D.D., Rueckert, D.: Simultaneous multi-scale registration using large deformation diffeomorphic metric mapping. IEEE Transactions on Medical Imaging 30(10), 1746-1759 (2011)

9. Dhollander, T., Veraart, J., Van Hecke, W., Maes, F., Sunaert, S., Sijbers, J., Suetens, P.: Feasibility and Advantages of Diffusion Weighted Imaging Atlas Construction in Q-Space. In: Fichtinger, G., Martel, A., Peters, T. (eds.) MICCAI 2011, Part II. LNCS, vol. 6892, pp. 166-173. Springer, Heidelberg (2011)

10. Vercauteren, T., Pennec, X., Perchant, A., Ayache, N.: Diffeomorphic demons: Efficient nonparametric image registration. NeuroImage 45(1), S61-S72 (2009)

11. Jupp, P., Mardia, K.: A unified view of the theory of directional statistics. International Statistical Review 57, 261-294 (1989)

12. Yap, P.T., Shen, D.: Spatial transformation of DWI data using non-negative sparse representation. IEEE Transactions on Medical Imaging (to appear)

13. Vialard, F.X., Risser, L., Rueckert, D., Cotter, C.: Diffeomorphic 3D image registration via geodesic shooting using an efficient adjoint calculation. International Journal of Computer Vision 97(2), 229-241 (2012) 